\section{Dasatinib-induced hemorrhagic colitis complicated with cytomegalovirus infection}

\author{
Aya Nakaya, Yoshiko Azuma, Shinya \\ Fujita, Atsushi Satake, Takahisa \\ Nakanishi, Yukie Tsubokura, Akiko \\ Konishi, Masaaki Hotta, Hideaki \\ Yoshimura, Kazuyoshi Ishii, Tomoki Ito, \\ Shosaku Nomura
}

First Department of Internal Medicine, Kansai Medical University, Osaka, Japan

\begin{abstract}
A 69-year-old man with chronic-phase chronic myeloid leukemia was initially treated with $100 \mathrm{mg}$ dasatinib once a day. Despite a major molecular response within 9 months, he developed hemorrhagic colitis 32 months after starting dasatinib. Colonoscopy identified multiple hemorrhagic ulcers in the transverse colon. The pathological findings indicated cytomegalovirus infection. Dasatinib was stopped and he was started on ganciclovir. Three months later, colonoscopy confirmed the disappearance of the hemorrhagic ulcers. Dasatinib is a second-generation tyrosine kinase inhibitor used to treat chronic myeloid leukemia. As a multi-kinase inhibitor that acts on SRC-family kinases, its broader off-target kinase-inhibitory activity may account for the adverse events of dasatinib. Although gastrointestinal bleeding is common in patients taking dasatinib, the combination of cytomegalovirus infection and hemorrhagic colitis in the absence of systemic immunodeficiency is rare. Based on this case of dasatinibinduced hemorrhagic colitis with cytomegalovirus infection, we describe a possible mechanism and effective treatment.
\end{abstract}

\section{Case Report}

A 69-year-old man with chronic-phase chronic myeloid leukemia (CML) was initially treated with $100 \mathrm{mg}$ dasatinib once a day. He achieved a complete hematological response within 1 month, a complete cytogenetic response within 6 months, and a major molecular response (MR) within 9 months. However, 10 months after starting dasatinib, he developed pleural effusion (PE) and was advised to stop dasatinib for 1 week. He was started on diuretics and the $\mathrm{PE}$ resolved immediately in response. $\mathrm{He}$ was then restarted on dasatinib but the dose was lowered to $50 \mathrm{mg}$ once a day. $\mathrm{He}$ retained the deep MR for 20 months even after the dose reduction. However, 32 months after starting dasatinib he developed hemorrhagic colitis (Figure 1). Colonoscopy revealed colon polyps and a polypectomy was planned for 3 months later. During those 3 months, he had intermittent hemorrhagic colitis. A repeat colonoscopy revealed multiple hemorrhagic ulcers in the transverse colon (Figure 2A). The pathological findings included the presence of cytomegalovirus (CMV) (Figure 2B), although his CMV7-HRP blood test was negative. Dasatinib therapy was stopped and he was started on ganciclovir. After a few weeks, he no longer had hemorrhagic colitis. Ganciclovir was withdrawn after 3 months. A colonoscopy confirmed disappearance of the hemorrhagic ulcers. He no longer receives anti-CML treatment and his deep MR has persisted.

\section{Discussion and Conclusions}

Dasatinib is a multi-kinase inhibitor that acts on SRC-family kinases, but also on BCR-ABL, c-KIT, EPHA2, and the PDGF receptor. ${ }^{1-3}$ This broader off-target kinaseinhibitory activity may account for the adverse effects of dasatinib, the most common of which are gastrointestinal symptoms, fluid retention, and skin rash. ${ }^{4}$

Gastrointestinal bleeding accounts for $\sim 20 \%$ of gastrointestinal symptoms in dasatinib patients, ${ }^{5}$ but there are few reports of an association between CMV infection and hemorrhagic colitis. ${ }^{6,7}$ Fluid retention is common in patients with $\mathrm{PE}$, but a paradoxical relationship exists between PE development and the efficacy of dasatinib. ${ }^{4}$

In our literature review of similar cases, there were two case reports in which CMV infection was associated with hemorrhagic colitis. One patient with chronic-phase CML was initially treated with imatinib but developed resistance and was switched to dasatinib (70 mg twice a day). ${ }^{6}$ Three years later, he developed hemorrhagic colitis, which was treated with steroid and mesalamine. While the hemorrhagic colitis temporarily improved, after steroid tapering it developed again. After dasatinib was stopped, the hemorrhagic colitis disappeared spontaneously within 7 days. Another patient with chronic-phase CML was initially treated with dasatinib (100 $\mathrm{mg}$ /day) and developed hemorrhagic colitis 30 months later. $^{7}$ Again, dasatinib was stopped immediately and the hemorrhagic colitis improved. After 6 weeks of ganci-
Correspondence: Aya Nakaya, First Department of Internal Medicine, Kansai Medical University, 2-5-1, Shin-machi, Hirakata, Osaka 573-1010, Japan.

Tel.: +81.72.804.2503

E-mail: nakaya1016@yahoo.co.jp

Key words: Dasatinib, Hemorrhagic colitis, Cytomegalovirus, Chronic myeloid leukemia.

Contribution: the authors contributed equally.

Conflict of interest: the authors declare no potential conflict of interest.

Received for publication: 20 September 2017. Accepted for publication: 9 October 2017

This work is licensed under a Creative Commons Attribution-NonCommercial 4.0 International License (CC BY-NC 4.0).

(C) Copyright A. Nakaya et al., 2017

Licensee PAGEPress, Italy

Hematology Reports 2017; 9:7415

doi:10.4081/hr.2017.7415

clovir treatment, the colonic mucosa was improved, as shown on colonoscopy. The pathological findings of both patients included CMV infection.

The mechanism underlying the development of hemorrhagic colitis is unknown. A previous report described infiltration of the colonic mucosa by CD $3+\mathrm{CD} 8+\mathrm{T}$ cells,${ }^{8}$ which suggested the involvement of an immune reaction in its pathogenesis. PE has also been attributed to an immunological reaction, because of the high lymphocyte frequency in pleural fluids and tissues. These associations suggest a common mechanism in hemorrhagic colitis and PE. Peripheral lymphocytosis precedes PE, as also occurs in hemorrhagic colitis. ${ }^{9}$ Dasatinib-induced complications developed in a marked proportion of patients with large granular lymphocyte (LGL) expansion, suggesting a pathogenic role for LGL in bleeding related to dasatinib. 8,10

The relationship between dasatinib and CMV infection is unknown. Dasatinib was shown to suppress the function of natural killer cells and $\mathrm{T}$ cells, by inhibiting SRCfamily kinases. ${ }^{11,12}$ Therefore, dasatinib may decrease immune tolerance, reducing the number of immunoregulatory cells and inhibiting signal transduction pathways. Furthermore, as dasatinib is an oral agent and is eliminated in the feces, it might cause local immune compromise and thus the reactivation of $\mathrm{CMV}$, with the lower gastrointestinal tract accordingly vulnerable, including to the development of hemorrhagic colitis. ${ }^{5}$ 


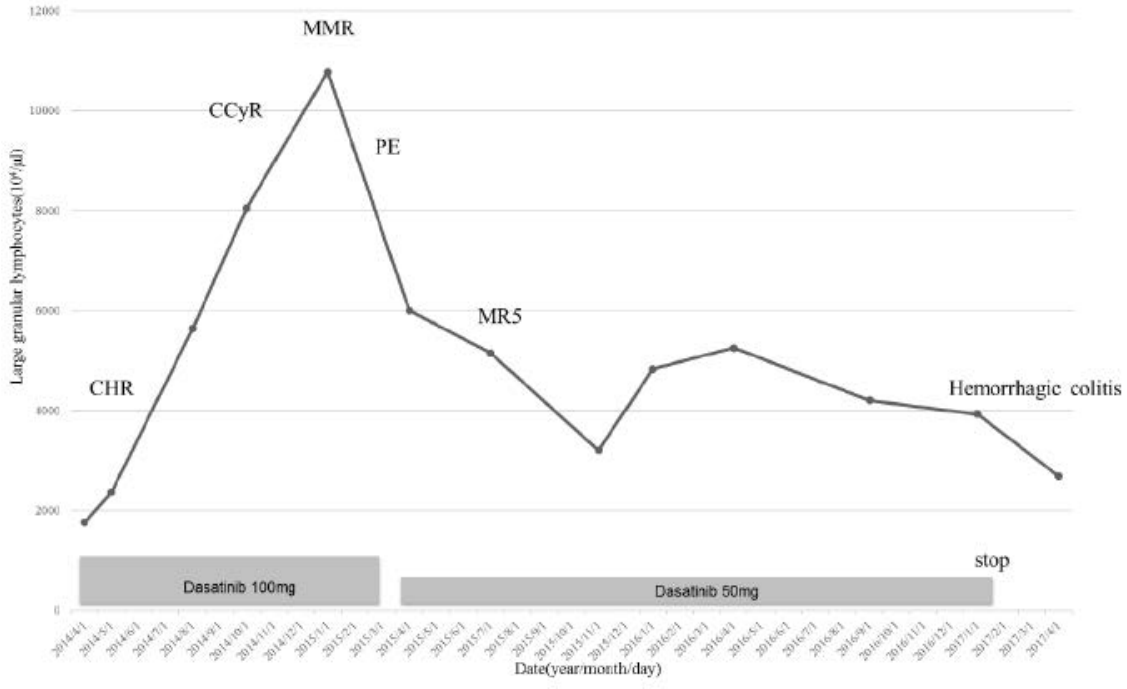

Figure 1. Patient's clinical course. After treatment with dasatinib for chronic myeloid leukemia, the patient had a complete hematological response (CHR) within 1 month, a complete cytogenetic response (CCyR) within 6 months, and a major molecular response (MMR) within 9 months. However, 10 months after starting dasatinib, he developed pleural effusion (PE) and dasatinib was discontinued. The PE resolved immediately. Dasatinib was restarted but at a lower dose of $50 \mathrm{mg}$ once a day. He retained the molecular response even after the dose reduction but he developed hemorrhagic colitis 32 months after starting dasatinib. Dasatinib was therefore stopped.

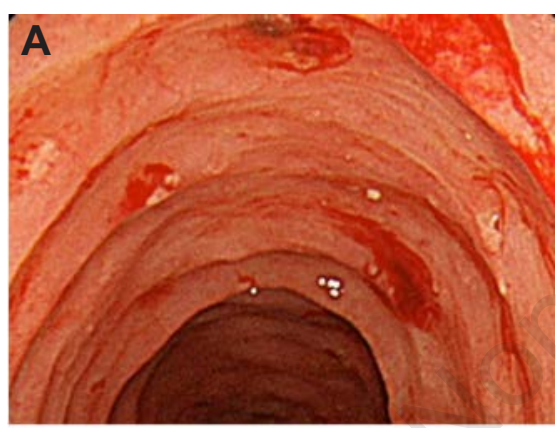

B

Figure 2. A) Endoscopic image demonstrating multiple mucosal hemorrhagic ulcers in the transverse colon. B) Immunohistochemistry of the biopsy specimen shows its positivity to CMV antigen, demonstrated using a monoclonal antibody $(\times 100)$.
The treatment of hemorrhagic colitis has not been established. Steroids may ameliorate symptoms but recurrences following a dose reduction have been reported. ${ }^{6,13}$ In patients with hemorrhagic colitis and CMV infection, steroids may be counterproductive and should be administered with caution. The efficacy of ganciclovir is not clear and in the treatment of hemorrhagic colitis has been invalidated, ${ }^{14}$ whereas the discontinuation of dasatinib was shown to be effective. ${ }^{15,16}$

Dasatinib-induced hemorrhagic colitis is relatively uncommon and further studies are needed to understand its mechanism. However, physicians should be aware of the risk of this adverse event in treating patients with dasatinib.

\section{References}

1. Lombardo LJ, Lee FY, Chen P, et al. Discovery of N-(2-chloro-6-methylphenyl)-2-(6-(4-(2-hydroxyethyl)piperazin-1-yl)-2-methylpyrimidin-4ylamino)thiazole-5-carboxamide (BMS-354825), a dual Src/Abl kinase inhibitor with potent antitumor activity in preclinical assays. J Med Chem
2004;47:6658-61.

2. Fabian MA, Biggs WH 3rd, Treiber DK, et al. A small molecule-kinase interaction map for clinical kinase inhibitors. Nat Biotechnol 2005;23:32936.

3. Rix U, Hantschel O, Dürnberger G, et al. Chemical proteomic profiles of the BCR-ABL inhibitors imatinib, nilotinib, and dasatinib reveal novel kinase and nonkinase targets. Blood 2007;110: 4055-63.

4. Eskazan AE, Eyice D, Kurt EA, et al. Chronic myeloid leukemia patients who develop grade I/II pleural effusion under second-line dasatinib have better responses and outcomes than patients without pleural effusion. Leuk Res 2014;38:781-7.

5. Quintás-Cardama A, Kantarjian H, Ravandi F, et al. Bleeding diathesis in patients with chronic myelogenous leukemia receiving dasatinib therapy. Cancer 2009;115:2482-90.

6. Patodi N, Sagar N, Rudzki Z, et al. Haemorrhagic colitis caused by dasatinib. Case Rep Hematol 2012; 2012:417106.

7. Yassin MA, Nashwan AJ, Soliman AT, et al. Cytomegalovirus-induced hemorrhagic colitis in a patient with chronic myeloid leukemia (chronic phase) on dasatinib as an upfront therapy. Clin Med Insights Case Rep 2015;8:77-81.

8. Mustjoki S, Ekblom M, Arstila TP, et al. Clonal expansion of T/NK-cells during tyrosine kinase inhibitor dasatinib therapy. Leukemia 2009;23:1398-405.

9. Kreutzman A, Juvonen V, Kairisto V, et al. Mono/oligoclonal $\mathrm{T}$ and NK cells are common in chronic myeloid leukemia patients at diagnosis and expand during dasatinib therapy. Blood 2010;116:77282.

10. Nagata Y, Ohashi K, Fukuda S, et al. Clinical features of dasatinib-induced large granular lymphocytosis and pleural effusion. Int J Hematol 2010;91: 799-807.

11. Blake SJ, Bruce Lyons A, et al. Dasatinib suppresses in vitro natural killer cell cytotoxicity. Blood 2008;111:4415-6.

12. Schade AE, Schieven GL, Townsend R, et al. Dasatinib, a small-molecule protein tyrosine kinase inhibitor, inhibits Tcell activation and proliferation. Blood 2008;111:1366-77.

13. Shimokaze T, Mitsui T, Takeda H, et al. Severe hemorrhagic colitis caused by dasatinib in Philadelphia chromosomepositive acute lymphoblastic leukemia. Pediatr Hematol Oncol 2009;26:44853. 
14. Sunami Y, Sato E, Ichikawa K, et al. Hemorrhagic colitis caused by dasatinib following cytomegalovirus enterocolitis in a patient with chronic myelogenous leukemia in the second chronic phase.
Rinsho Ketsueki 2011;52:282-6.

15. Ono Y, Mori T, Kato J, et al. Hemorrhagic colonic ulcers caused by dasatinib for chronic myelogenous leukemia. Int J Hematol 2010;92:556-8.
16. Erkut M, Erkut N, Ersoz S, et al. A case of acute colitis with severe rectal bleeding in a patient with chronic myeloid leukemia after dasatinib use. Acta Haematol 2010;123:205-6. 